\title{
Deep Learning for the Automation of Particle Analysis in Catalyst Layers for Polymer Electrolyte Fuel Cells
}

\author{
André Colliard-Granero ${ }^{\mathrm{a}, \mathrm{b}}$, Mariah Batoolc, Jasna Jankovic ${ }^{\mathrm{c}}$, Jenia Jitsev ${ }^{\mathrm{d}}$, \\ Michael H. Eikerling ${ }^{\mathrm{a}, \mathrm{e}}$, Kourosh Malek ${ }^{\mathrm{a}, *}$, Mohammad J. Eslamibidgoli ${ }^{\mathrm{a}, *}$ \\ ${ }^{a}$ Institute of Energy and Climate Research, Theory and Computation of Energy Materials \\ (IEK-13), Forschungszentrum Jülich, 52425 Jülich, Germany \\ ${ }^{b}$ Department of Chemistry, University of Cologne, Greinstr. 4-6, 50939 Cologne, Germany \\ ${ }^{c}$ Department of Materials Science and Engineering, University of Connecticut, 97 \\ NorthEagleville Road, Unit 3136, Storrs, CT 06269-3136, USA \\ ${ }^{d}$ Jülich Supercomputing Center, Forschungszentrum Jülich, 52425 Jülich, Germany \\ ${ }^{e}$ Chair of Theory and Computation of Energy Materials, Faculty of Georesources and \\ Materials Engineering, RWTH Aachen University, Aachen 52062, Germany
}

\begin{abstract}
The rapidly growing use of imaging infrastructure in the energy materials domain drives significant data accumulation in terms of their amount and complexity. The applications of routine techniques for image processing in materials research are often ad hoc, indiscriminate, and empirical, which renders the crucial task of obtaining reliable metrics for quantifications obscure. Moreover, these techniques are expensive, slow, and often involve several preprocessing steps. This paper presents a novel deep learning-based approach for the high-throughput analysis of the particle size distributions from transmission electron microscopy (TEM) images of carbon-supported catalysts for polymer electrolyte fuel cells. Our approach employs training an instance segmentation model, called StarDist [Schmidt et al. Medical Image Computing and Computer-Assisted Intervention - MICCAI 2018, Lecture Notes in Computer Science, vol 11071. Springer, Cham], which resolves the main challenge in the pixel-wise localization of nanoparticles in TEM images: the overlapping particles. The segmentation maps outperform similar models reported in the lit-
\end{abstract}

\footnotetext{
* Corresponding author

Email addresses: k.malek@fz-juelich.de (Kourosh Malek), m.eslamibidgoli@fz-juelich.de (Mohammad J. Eslamibidgoli)
} 
erature, and the results on particle size analyses agree well with the manual particle size measurements, albeit at a significantly lower cost.

Keywords: High Throughput Screening, Catalyst Layers, Fuel Cells, Microscopy Characterization, Deep Learning, Convolutional Neural Networks

\section{Introduction}

Relentless exhaustion of non-renewable resources in combination with ever-rising environmental concerns demand escalating efforts to develop and improve clean energy technology [1, 2, 3. As one of the leading candidates for clean energy

5 applications, especially in the automotive sector, polymer electrolyte membrane fuel cells (PEMFCs) demonstrate remarkable technical characteristics such as high power density, high energy conversion efficiency, instant start-up/shutdown and zero-emission operation [4, 5, 6]. Despite numerous advantages, PEMFC technology suffers from a significant drawback in terms of high costs and insufficient performance of the catalyst layer (CL). The latter is fabricated using expensive Platinum (Pt) nanoparticles (NPs), which are supported on a high-surface area carbon material $(\mathrm{Pt} / \mathrm{C})$ [7, 8. Therefore, further advances in performance and cost reduction of the Pt-based catalyst layer are vital to ensure the widespread deployment of PEMFCs [9, 10, 11.

15 In the past three decades, a plethora of research activities have focused on developing and evaluating novel alternatives to improve the performance of Pt-based CL through experimental methods, computational studies, and comprehensive characterization 12, 13, 14, 15, 16, 17, 18, 19, 20, 21, 22. High-resolution X-ray and electron microscopy (in situ/ex situ) characterization methods are increasingly used for the evaluation of the catalytic activity and stability of the catalyst layer. Examples include ultra-small angle X-ray scattering (u-SAXS), focussed ion beam scanning electron microscopy (FIB-SEM), transmission electron microscopy (TEM), scanning transmission electron microscopy (STEM), or scanning transmission X-ray microscopy (STXM) [23, 24, 25, 26].

${ }_{25}$ To unravel the connection between microstructure properties and performance, 
it becomes essential to quantify certain features of the imaging data correctly, e.g., the particle size distribution (PSD), pore size, pore network, or aggregate size - with less reliance on human intervention and interpretation [27, 28. This quantification may require additional analyses, which can be done either manually by the operator or automatically via processing the images through a set of specialized algorithms to extract the pertinent structural features [29, 30]. The type of post-processing analysis of materials characterization data alluded to be beneficial as numerous studies suggested that the electrochemical performance of CL is strongly dependent on the surface area, size, shape, and distribution of Pt NPs 31, 32, 33, 34. For example, an increase in mean Pt particle size in an aged cathode and subsequent reduction at anode, associated with $\mathrm{Pt}^{2+}$ ion crossover, could indicate degradation of the carbon support [35, 36, 37]. In another study the larger particle size and agglomeration after potential loading was attributed to the dissolution and re-deposition of $\mathrm{Pt}$ in the catalyst

40 layer 38. Smaller Pt particles are not favorable either because of the stronger adsorption of reaction intermediates at the edges and corners of NPs; the latter results in blockage of oxygen reduction reaction path and makes the $\mathrm{Pt}$ more susceptible to dissolution [39].

The widely employed technique for particle size analysis entails measuring a 45 set of manually drawn straight lines from the edges of individual particles in the image [40]. This approach employs standard image processing tools like ImageJ [41] and may requires thresholding the image and applying filters for denoising, to segment the particles from the support material or background. Notwithstanding, this essential thresholding step becomes tricky in capturing small particles or when the contrast between particle and support material is not strong. In addition, in a one-at-a-time inspection of particles, the operator usually considers only a subset of the particles for each image, resulting in the loss of information. The long, tedious, and detailed manual procedure makes this approach ineffective for high-throughput or real-time analysis. Moreover, ${ }_{55}$ identifying the overlapping particles is strongly dependent on the operator's bias and judgment. These issues generate an unreliable metric for the quantification 
and can slow down or even falsify the analysis.

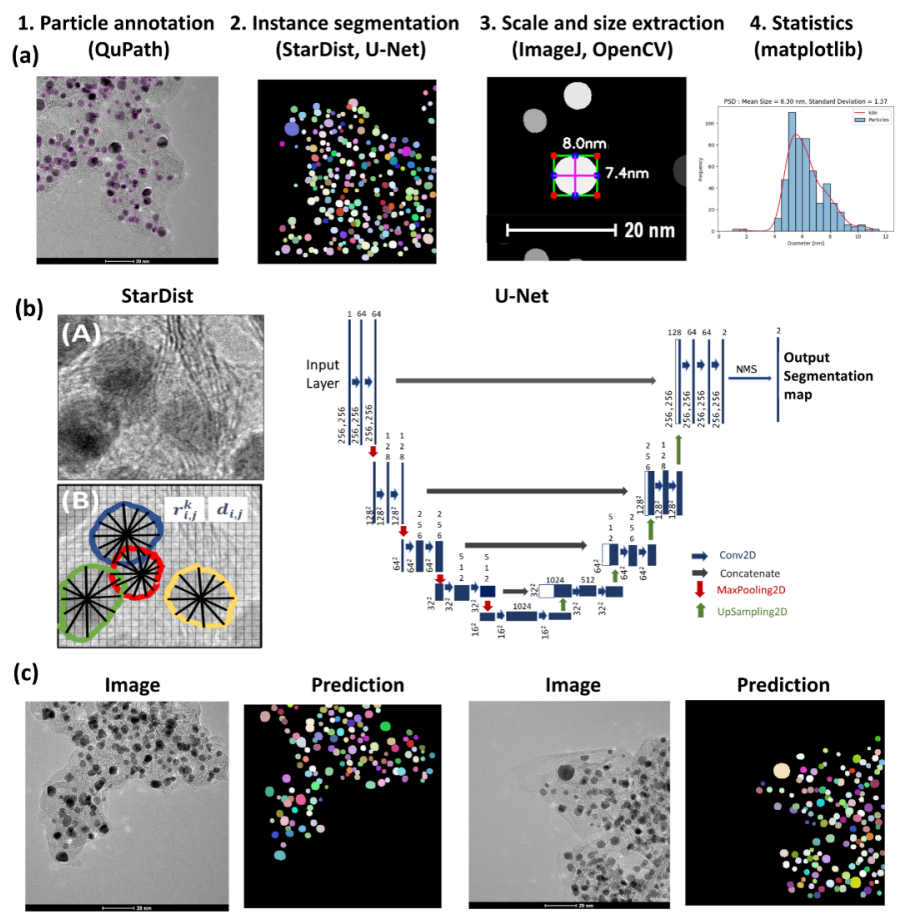

Figure 1: (a) Methodical pipeline for deep learning-based analysis of particle size distribution involving 1. particle annotation, 2. supervised training for the instance segmentation of particles, 3. setting scale for image calibration and particle size extraction with computer vision implemented using OpenCV library [42, 4. Obtaining particle size statistics with visualization libraries, e.g., matplotlib. (b) The left panel shows a TEM image with overlapped Pt particles supported on a carbon agglomerate; the StarDist method used in this work predicts star-convex polygons parameterized by the radial distances ri,j, and object probabilities di,j. for the pixel i,j 43]. The right panel shows the U-Net architecture 44] and selected the final instances via the non-maximum suppression (NMS) technique. (c) Example TEM images and their corresponding predicted instance segmentation of Pt particles using StarDist and U-Net.

Semi-automated approaches for the analysis of well-separated and monodisperse NPs have been developed. For example, for magnetite NPs, classical approaches using Otsu's binarization with Canny edge detection and edge linking algorithms were employed [45]. For the population analysis of Pt NPs on glassy Carbon, an earlier report has introduced the local adaptive threshold (LAT) in the im- 
age processing steps [46]. This step improved the segmentation of particles by thresholding the smaller patches of the image, but at the cost of additional computing time [46]. Ponti and co-workers developed PEBBLES [47, the software program for the size measurement and morphological analysis of nanoparticles in TEM micrographs. PEBBLES applies fitting 3D intensity models to relate the contrast of the image and the depth of the dip in the intensity surface to the NP size, making the automatic measurement and analysis of the distribution of morphological parameters passible. However, as pointed out by the authors, the software is limited in accurately fitting the overlapping NPs or the heterostructured NPs (e.g., core-shell NPs) [47.

Over the last decade, deep learning (DL) methods based on the convolutional neural networks (ConvNets) have transformed field of computer vision by out75 performing the conventional methods in various image and video recognition tasks such as in verification/identification, classification, object detection, and segmentation, as well in other applications including image reconstruction, denoising, image synthesis, colorization, style transfer and other tasks [48, 49. To leverage the advancements in DL-based approaches in microscopy image processing, more recently, the ConvNets algorithms have been deployed to several platforms for various analysis tasks like segmentation, object detection, denoising, or super-resolution microscopy. The most exciting example is the recently developed ZeroCostDL4Mic [50] platform that provides the advantage of free computational resources on GoogleColab for training the deep neural network models. Unlike the conventional procedures requiring several preprocessing steps for particle analysis to construct suitable features, DL models utilize the information content directly from the raw TEM micrographs [51]; once a suitable model is trained on a representative dataset, they can predict swiftly and accurately without the necessity for the human-based alteration of param90 eters. Moreover, the robustness of DL models is validated with various metrics like precision, recall, or accuracy, which introduces well-defined quantification metrics for particle analysis.

More recently, Yao et al. implemented the popular U-Net model for real-time 
nanoparticle segmentation in liquid-phase TEM videos [52]. This novel approach enabled the statistical analysis of the diffusion, reaction, and assembly kinetics of the rod-, prism- and cube-shaped colloidal NPs 52. Cole and co-workers introduced ImageDataExtractor [53, 54], a novel tool to automate the extraction and analysis of microscopy imaging data from the scientific literature. The main feature of the ImageDataExtractor is its capability in segmentation and quantification of NPs via Bayesian DL for a diverse set of morphologies [54. However, as will be discussed, ImageDataExtractor is limited in the accurate localization of overlapping NPs, as is the case for Pt NPs on a carbon support material.

This paper presents a novel DL-based approach to automate the PSD analy105 sis from TEM micrographs of catalyst layers in PEMFCs. We employed the pre-trained StarDist model [43, and fine-tuned it on our annotated imaging dataset for the instance segmentation of Pt NPs on the high surface area Carbon support. The trained model largely resolved the localization of overlapping NPs, and the proposed pipeline will enable the automation of PSD analysis. Moreover, unlike the conventional procedure based on image thresholding, this approach adopts definite quantification metrics for evaluating the segmentation model.

\section{Methods}

Samples for TEM imaging were either catalyst powders directly dispersed on the TEM grid or thin sections of the fuel cell electrodes. The catalyst powders were prepared by dispersing catalyst powders in $50 \mathrm{vol} \%$ isopropyl alcohol in water and dropped on the 200 lacey carbon TEM grid. Thin sections of the electrodes were prepared by embedding the electrode in a 1:1 mixture of trimethylolpropane triglycidyl ether resin (Sigma-Aldrich, USA) and 4,4'-methylenebis (2-methylcyclohexylamine, Sigma-Aldrich, USA) hardener, and sectioning thin slices $(\approx 100 \mathrm{~nm})$ using Leica UCT ultramicrotome and Ultra $45^{\circ}$ DiATOME knife, USA. The sections were placed on 200 mesh $\mathrm{Cu}$ TEM grids. Images for 
particle size distribution were obtained using Talos F200X scanning transmission electron microscope (Thermo Fisher Scientific, USA) with electron accelerating voltage of $200 \mathrm{kV}$.

Our DL-based approach for the PSD analysis involves the four steps in Figure 1(a): (1) manual annotation of Pt NPs, (2) supervised learning for particle segmentation, (3) automated diameter measurement on the predicted region-ofinterest (ROI), and (4) statistics and visualization. The first step for the supervised learning task of segmentation is the pixel-wise annotation of NPs in the TEM images. The quality of the trained model largely depends on the quality of the labeled data; therefore, it is important to choose an efficient and accurate tool for this labor-intensive step. For this purpose, we used QuPath [55], a user-friendly open-source software initially developed for quantitative pathology and bioimage analysis. Forty high-resolution TEM images $(1024 \times 1024$ and $2048 \times 2048$ pixels) of Pt NPs on Carbon support were manually annotated, with 34 used for training purposes and 6 for validation purposes. The image set comprised four different magnification levels, at 10, 20, 50, and $100 \mathrm{~nm}$ scales, providing a generalization of the scale range for the training of more robust models. An expert performed the annotations considering visual evidence for the overlapping particles while eluding the strongly crowded Pt aggregates. We make this dataset publicly available.
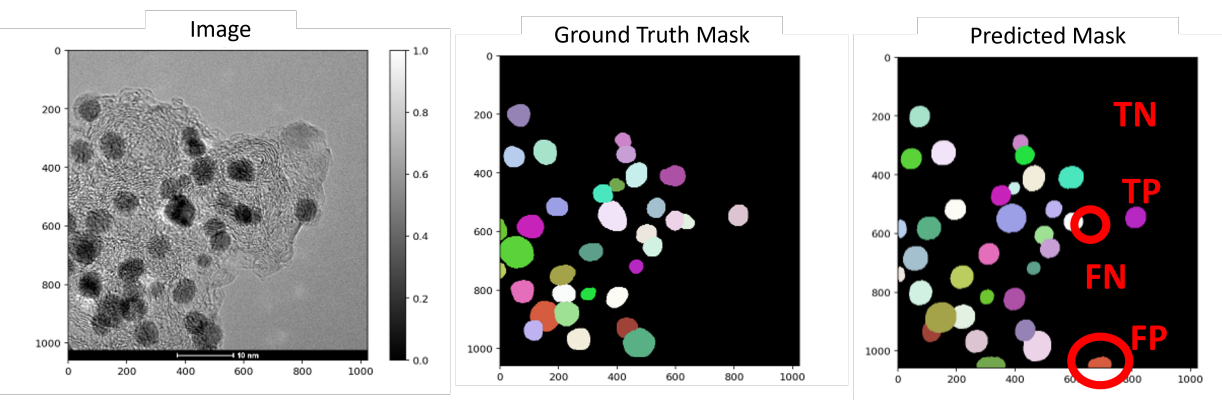

Figure 2: Demonstration of a TEM image along with the ground truth and predicted masks. Examples of true-positive (TP), true-negative (TN), false-positive (FP), and false-negative $(\mathrm{FN})$ predictions are shown on the predicted mask. 
Given the images and corresponding labeled masks as ground truth, for the pixel-level localization of individual Pt particles, we trained the StarDist model [43. StarDist employs the U-Net architecture [4] and overcomes the typical segmentation errors for the dense prediction of merged bordering particles by localizing the singular particles as star-convex polygons (Figure 1 (b)). StarDist was initially developed for the segmentation of biological systems to differentiate individual overlapping cells in crowded images. In this model, each pixel is parameterized by two values, the radial distances and object probabilities. For the former, a star-convex polygon is fitted from the pixel position to the edges of the localized particle. On the other hand, object probability is represented by the normalized shortest distance from the edge of the particle; the latter increases with the decreasing distance of the particle center.

155 Standard architectures for image segmentation usually involve fully convolutional networks that first perform convolution and down-sampling operations to extract essential features from the image (encoding into a latent space), followed by up-sampling and transpose convolution operations that make the activation maps larger until it reaches the input image size (decoding from a latent space). In the U-Net architecture, in addition to this data stream, the up-sampled decoder features are concatenated with the exact corresponding resolution from encoder feature maps (as shown by the gray arrows in Figure 1 (b)). Concatenating encoder and decoder feature maps enables recognizing object boundaries or edges by combining features across all levels, from low to high, leading to more accurate output segmentation maps (Figure 1 (c)). The StarDist implementation of U-Net employs data augmentation to expand the size of the training set by generating modified versions of images in the dataset, where the images are cropped in patches and augmented by rotation, changing intensity, and Gaussian blurring. In this model, the non-maximum suppression (NMS) technique is further used to suppress the multiple detections of individual objects. This way, the predicted box with higher object probability suppresses the overlapped boxes with lower probabilities. The number of epochs and the number of steps per epoch for training were chosen as 400 and 100, respectively. 

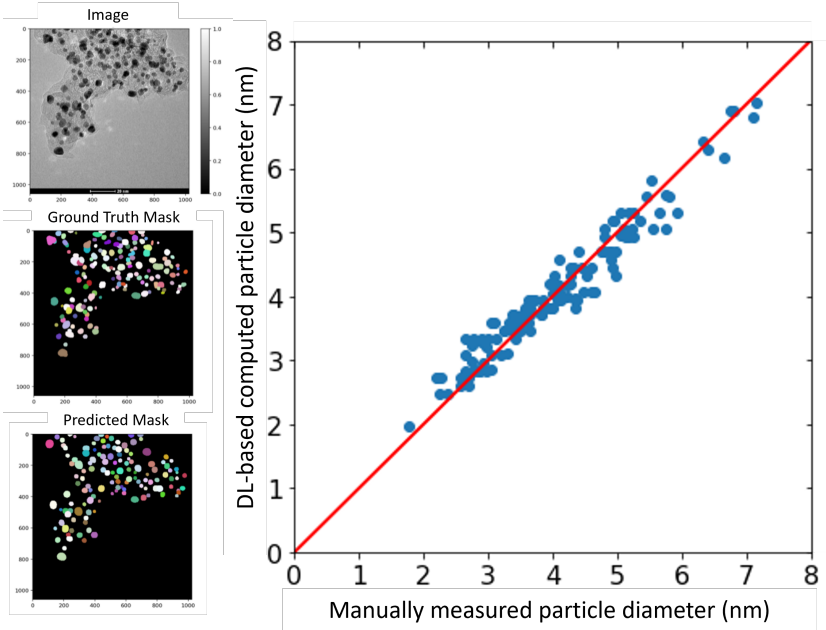

Figure 3: The left panel shows the TEM image, ground truth mask, and predicted mask. The right panel compares the manually measured particle diameter with the deep learning-based computed particle diameter.

The performance of the trained model was analyzed by plotting standard metrics concerning the Intersection over Union (IoU) threshold. The IoU measures the number of pixels common between the ground truth and prediction masks divided by the total number of existing pixels in both masks. Conventionally, reliable predictions correspond to an IoU value $\geq 0.5$. In our case, a true-positive (TP) represents the case if a prediction-target mask pair for nanoparticles has an IoU score that exceeds 0.5 ; likewise, a true-negative $(\mathrm{TN})$ is that for support or background. On the other hand, a false-positive (FP) indicates a predicted NP mask with no associated ground truth mask, and a false-negative (FN) indicates a ground truth NP mask with no associated predicted mask. Examples of TP, TN, FP, and FN are demonstrated in Figure 2. This way, the metrics of accuracy, precision, recall, and F1 score are defined as,

$$
\begin{gathered}
\text { Accuracy }=(T P+T N) /(T P+F P+T N+F N) ; \\
\text { Precision }=(T P) /(T P+F P) ; \\
\text { Recall }=(T P) /(T P+F N) ;
\end{gathered}
$$




$$
F 1=2 \times \text { Precision } \times \text { Recall } /(\text { Precision }+ \text { Recall })
$$

For an image with many NPs, it is not a severe problem to have a few FN predictions because it does not significantly affect the statistical analysis. However, a model with high precision indicates false particles are not introduced to the particle size distribution analysis. Therefore, precision is a reliable measure for the evaluation of the model performance. The accuracy, on the other hand, can be a misleading metric for the images with a small number of NPs as it becomes biased in mainly relating how well the model predicts the background.

Given the model's prediction for segmentation maps, the ROI for each particle was white-filled, cropped, and saved into a JPEG file. Next, using the contrasted contours, we used the OpenCV library [42] to place a bounding box around the predicted masks and measured the number of pixels along with the $\mathrm{X}$ or $\mathrm{Y}$ directions (step 3 in Figure 1 (a)). Here, manual image calibration was used for a pixel-to-real-distance conversion. Lastly, The particle size can be estimated as the average values along with the $\mathrm{X}$ and $\mathrm{Y}$ directions, and the corresponding histogram can be generated for the PSD analysis (step 4 in Figure 1 (a)).

\section{Results and Discussion}

Figure 3 compares the manually measured particle diameter performed by an expert as a reference and the computed particle diameter values for a test TEM image according to the approach presented. The image is shown in the left panel along with the ground truth and predicted segmentation masks. The strong correlation between the values indicates the effectiveness of our deep learning-based approach for the high-throughput particle size analysis with minimal human interventions. For this image, the fine-tuned StarDist model on our annotated dataset could localize a total of 134 particles out of the 150 particles in the ground truth mask. Interestingly enough, the model captured most of the overlapping particles. The obtained accuracy, precision, recall and F1-score at an IoU threshold of 0.5 were $71 \%, 0.86 \%, 0.81 \%, 0.83 \%$, respectively. Even though the performance of the model can be further improved by expanding the train- 
(a)

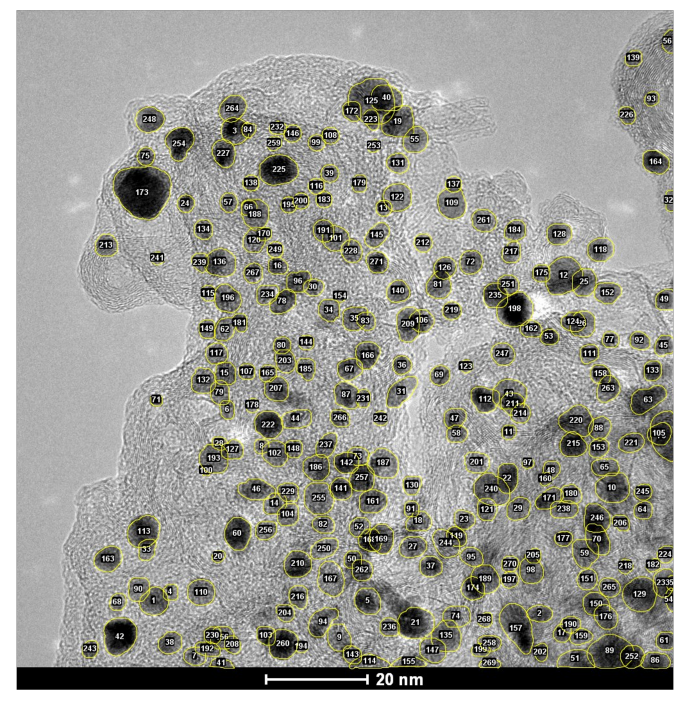

(b)

TEM Image
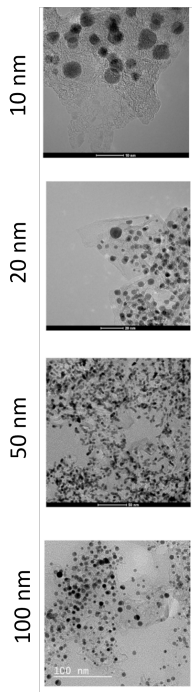

StarDist Image Data

(this work) Extractor 2.0

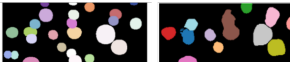
$\because 0$ 8: 0
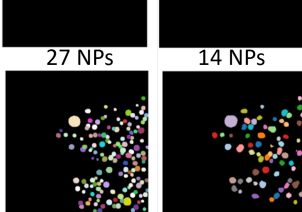

$144 \mathrm{NPS}$ $67 \mathrm{NPS}$

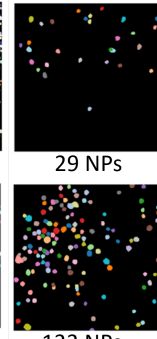

133 NPs

Figure 4: (a) Localization and counting the particles made available by the deep learning approach. (b) Comparison between the predicted segmentation maps of test images at different scales using the StarDist model employed in this work and the ImageDataExtractor 2.0 54.

ing set, the main origin of discrepancies is for the non-rounded shape particles.

This can be explained by general difficulty to predict non-convex shapes that is rooted in StarDist radial model for contour representation. As can also be seen from the predicted map, most of the localized particles are round-shaped or have a convex contour. This would suggest further room for improvements in the correct shape predictions using for example algorithms that use a more flexible contour representation for learning like the Contour Proposal Networks $(\mathrm{CPN})$ in Ref. [56] or PolarMask++ [57].

Figure 4 (a) demonstrates another valuable capability of the deep learning approach to localize particles in the TEM images. The ROI data from the segmentation model makes it possible to assign a number and count the particles in the image. Likewise, with the ROI data, the area of individual particles can be calculated. These abilities can be in particular helpful in estimating the $\mathrm{Pt} / \mathrm{C}$ ratio or the $\mathrm{Pt}$ mass loading from the image. Figure 4 (b) compares the 
segmentation maps for the test images obtained in this study at different scales with those from recently developed ImageDataExtractor 2.0 software [54. The ImageDataExtractor model also uses a deep learning approach for microscopy particle analysis. For our dataset, the StarDist model significantly outperforms the ImageDataExtractor 2.0 in predicting the number of particles and localizing the overlapping particles. As shown in Fig. 4 (b), for the test TEM images at 10, 20, 50, and $100 \mathrm{~nm}$ scales, the StarDist predicted 27, 144, 628, and 286 particles, respectively, while the existing model found only 14, 67, 29, and 133 particles. Notably, in ImageDataExtractor 2.0, the lack of capability to separate the overlapping particles leads to a fusion of the near particles in segmentation masks, reducing the total amount of detected particles.

Lastly, with the computed particle sizes the histograms for particle size distributions can be generated using typical visualization packages available in various programming languages. An example is shown in Fig. 5. Here, we used a bin size of $0.5 \mathrm{~nm}$ and the kernel density estimation (KDE) function for fitting the histogram. Binning can also be accomplished by using conventional statistical methods and expressions including Sturge's rule, Doane's Rule, Scott's Rule, Rice's Rule or Freedman and Diaconis's rule [58, 59, 60, 61, 62. Fitting for particle size distribution histogram can be performed using algorithms based on a variety of distribution functions such as polynomial distribution, Gaussian distribution, Weibull probability distribution, lognormal distribution, or Rayleigh distribution. 63, 64, 65, 66.
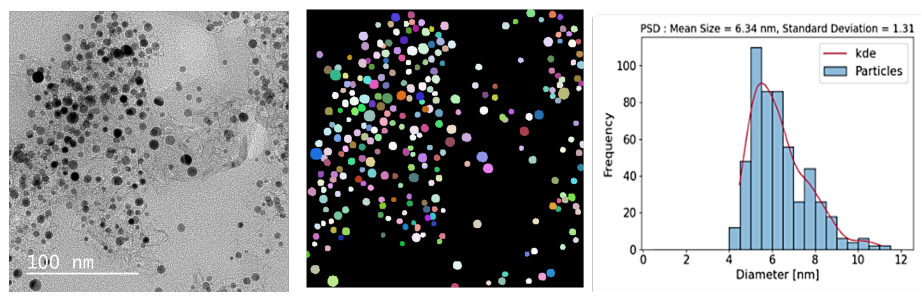

Figure 5: Particle size distribution was obtained using the deep learning-based approach of the instance segmentation of nanoparticles in the TEM images of Pt/C catalyst layers. 
In conclusion, this paper presented a deep learning-based approach to automating particle size analysis in the TEM images of catalyst layers for polymer electrolyte fuel cells. The pre-trained StarDist model, fine-tuned on our annotated imaging dataset, could segment round-shaped overlapping nanoparticle

The authors acknowledge the financial support from the Federal Ministry of Science and Education (BMBF) under the German-Canadian Materials Accelera- 
tion Centre (GC-MAC) grant number 01DM21001A. The authors also gratefully acknowledge the Gauss Centre for Supercomputing e.V. (www.gauss-centre.eu) for funding this project by providing computing time through the John von Neumann Institute for Computing (NIC) on the GCS Supercomputer JUWELS [72] at Jülich Supercomputing Centre (JSC).

\section{References}

[1] J. Nowotny, J. Dodson, S. Fiechter, T. M. Gür, B. Kennedy, W. Macyk, T. Bak, W. Sigmund, M. Yamawaki, K. A. Rahman, Towards global sustainability: Education on environmentally clean energy technologies, Renewable and Sustainable Energy Reviews 81 (2018) 2541-2551.

[2] I. Staffell, D. Scamman, A. V. Abad, P. Balcombe, P. E. Dodds, P. Ekins, N. Shah, K. R. Ward, The role of hydrogen and fuel cells in the global energy system, Energy \& Environmental Science 12 (2) (2019) 463-491.

[3] D. K. Niakolas, M. Daletou, S. G. Neophytides, C. G. Vayenas, Fuel cells are a commercially viable alternative for the production of "clean" energy, Ambio 45 (1) (2016) 32-37.

[4] J. Zhang, H. Zhang, J. Wu, J. Zhang, Chapter 1-pem fuel cell fundamentals, PEM Fuel Cell Testing and Diagnosis (2013) 1-42.

[5] F. T. Wagner, B. Lakshmanan, M. F. Mathias, Electrochemistry and the future of the automobile, The Journal of Physical Chemistry Letters 1 (14) (2010) 2204-2219.

[6] A. Ajanovic, R. Haas, Prospects and impediments for hydrogen and fuel cell vehicles in the transport sector, International Journal of Hydrogen Energy 46 (16) (2021) 10049-10058.

[7] K. Sopian, W. R. W. Daud, Challenges and future developments in proton exchange membrane fuel cells, Renewable energy 31 (5) (2006) 719-727. 
[8] J. Zhang, PEM fuel cell electrocatalysts and catalyst layers: fundamentals and applications, Springer Science \& Business Media, 2008.

[9] J. Chen, Z. Ou, H. Chen, S. Song, K. Wang, Y. Wang, Recent developments of nanocarbon based supports for pemfcs electrocatalysts, Chinese Journal of Catalysis 42 (8) (2021) 1297-1326.

[10] M. K. Debe, Electrocatalyst approaches and challenges for automotive fuel cells, Nature 486 (7401) (2012) 43-51.

[11] M. J. Eslamibidgoli, J. Huang, T. Kadyk, A. Malek, M. Eikerling, How theory and simulation can drive fuel cell electrocatalysis, Nano Energy 29 (2016) 334-361.

[12] M. Eikerling, A. A. Kornyshev, A. A. Kulikovsky, Physical modeling of fuel cells and their components, Wiley Online Library, 2007.

[13] Y. He, Q. Tan, L. Lu, J. Sokolowski, G. Wu, Metal-nitrogen-carbon catalysts for oxygen reduction in pem fuel cells: self-template synthesis approach to enhancing catalytic activity and stability, Electrochemical Energy Reviews 2 (2) (2019) 231-251.

[14] P. Spinelli, C. Francia, E. Ambrosio, M. Lucariello, Semi-empirical evaluation of pemfc electro-catalytic activity, Journal of Power Sources 178 (2) (2008) 517-524.

[15] M. Sabharwal, L. M. Pant, N. Patel, M. Secanell, Computational analysis of gas transport in fuel cell catalyst layer under dry and partially saturated conditions, Journal of The Electrochemical Society 166 (7) (2019) F3065.

[16] S. Jomori, K. Komatsubara, N. Nonoyama, M. Kato, T. Yoshida, An experimental study of the effects of operational history on activity changes in a pemfc, Journal of The Electrochemical Society 160 (9) (2013) F1067.

[17] Q. Wang, M. Eikerling, D. Song, Z. Liu, T. Navessin, Z. Xie, S. Holdcroft, Functionally graded cathode catalyst layers for polymer electrolyte fuel 
cells: I. theoretical modeling, Journal of the Electrochemical Society 151 (7) (2004) A950.

[18] K. Malek, T. Mashio, M. Eikerling, Microstructure of catalyst layers in pem fuel cells redefined: a computational approach, Electrocatalysis 2 (2) (2011) 141-157.

[19] M. Eikerling, A. Kulikovsky, Polymer electrolyte fuel cells: physical principles of materials and operation, CRC Press, 2014.

[20] E. Sadeghi, A. Putz, M. Eikerling, Hierarchical model of reaction rate distributions and effectiveness factors in catalyst layers of polymer electrolyte fuel cells, Journal of the Electrochemical Society 160 (10) (2013) F1159.

[21] T. Muzaffar, T. Kadyk, M. Eikerling, Tipping water balance and the pt loading effect in polymer electrolyte fuel cells: a model-based analysis, Sustainable Energy \& Fuels 2 (6) (2018) 1189-1196.

[22] J. P. Owejan, J. E. Owejan, W. Gu, Impact of platinum loading and catalyst layer structure on pemfc performance, Journal of The Electrochemical Society 160 (8) (2013) F824.

[23] W. Li, Z. Wang, F. Zhao, M. Li, X. Gao, Y. Zhao, J. Wang, J. Zhou, Y. Hu, Q. Xiao, et al., Phosphorene degradation: visualization and quantification of nanoscale phase evolution by scanning transmission x-ray microscopy, Chemistry of Materials 32 (3) (2020) 1272-1280.

[24] A. P. Hitchcock, V. Lee, J. Wu, M. M. West, G. Cooper, V. Berejnov, T. Soboleva, D. Susac, J. Stumper, Characterizing automotive fuel cell materials by soft x-ray scanning transmission x-ray microscopy, in: AIP Conference Proceedings, Vol. 1696, AIP Publishing LLC, 2016, p. 020012.

[25] K. Karan, Pefc catalyst layer: Recent advances in materials, microstructural characterization, and modeling, Current Opinion in Electrochemistry 5 (1) (2017) 27-35. 
[26] R. Singh, A. Akhgar, P. Sui, K. Lange, N. Djilali, Dual-beam fib/sem characterization, statistical reconstruction, and pore scale modeling of a pemfc catalyst layer, Journal of The Electrochemical Society 161 (4) (2014) F415.

[27] J.-N. Rouzaud, C. Clinard, Quantitative high-resolution transmission electron microscopy: a promising tool for carbon materials characterization, Fuel Processing Technology 77 (2002) 229-235.

[28] A. Abbas, G. Fathifazl, B. Fournier, O. B. Isgor, R. Zavadil, A. G. Razaqpur, S. Foo, Quantification of the residual mortar content in recycled concrete aggregates by image analysis, Materials characterization 60 (7) (2009) 716-728.

[29] L. Wojnar, Image analysis: applications in materials engineering, Crc Press, 2019.

[30] K. M. Halsall, V. M. Ellingsen, J. Asplund, R. H. Bradshaw, M. Ohlson, Fossil charcoal quantification using manual and image analysis approaches, The Holocene 28 (8) (2018) 1345-1353.

[31] R. L. Borup, A. Kusoglu, K. C. Neyerlin, R. Mukundan, R. K. Ahluwalia, D. A. Cullen, K. L. More, A. Z. Weber, D. J. Myers, Recent developments in catalyst-related pem fuel cell durability, Current Opinion in Electrochemistry 21 (2020) 192-200.

[32] P. Jovanovič, A. Pavlišič, V. S. Šelih, M. Šala, N. Hodnik, M. Bele, S. Hočevar, M. Gaberšček, New insight into platinum dissolution from nanoparticulate platinum-based electrocatalysts using highly sensitive in situ concentration measurements, ChemCatChem 6 (2) (2014) 449-453.

[33] A. Pavlišič, P. Jovanovič, V. S. Šelih, M. Šala, N. Hodnik, M. Gaberšček, Platinum dissolution and redeposition from $\mathrm{pt} / \mathrm{c}$ fuel cell electrocatalyst at potential cycling, Journal of The Electrochemical Society 165 (6) (2018) F3161. 
[34] D. J. Myers, X. Wang, M. C. Smith, K. L. More, Potentiostatic and potential cycling dissolution of polycrystalline platinum and platinum nanoparticle fuel cell catalysts, Journal of The Electrochemical Society 165 (6) (2018) F3178.

[35] E. Guilminot, A. Corcella, F. Charlot, F. Maillard, M. Chatenet, Detection of pt $\mathrm{z}+$ ions and pt nanoparticles inside the membrane of a used pemfc, Journal of The Electrochemical Society 154 (1) (2006) B96.

[36] M. J. Eslamibidgoli, P.-É. A. Melchy, M. H. Eikerling, Modeling the local potential at pt nanoparticles in polymer electrolyte membranes, Physical Chemistry Chemical Physics 17 (15) (2015) 9802-9811.

[37] S. Helmly, M. Eslamibidgoli, K. A. Friedrich, M. Eikerling, Local impact of pt nanodeposits on ionomer decomposition in polymer electrolyte membranes, Electrocatalysis 8 (6) (2017) 501-508.

[38] Z.-M. Zhou, Z.-G. Shao, X.-P. Qin, X.-G. Chen, Z.-D. Wei, B.-L. Yi, Durability study of pt-pd/c as pemfc cathode catalyst, International Journal of Hydrogen Energy 35 (4) (2010) 1719-1726.

[39] M. Maciá, J. Campina, E. Herrero, J. Feliu, On the kinetics of oxygen reduction on platinum stepped surfaces in acidic media, Journal of Electroanalytical Chemistry 564 (2004) 141-150.

[40] A. Subcommittee, Astm e112-10 standard test methods for determining average grain size, ASTM, Philadelphia.

[41] C. A. Schneider, W. S. Rasband, K. W. Eliceiri, Nih image to imagej: 25 years of image analysis, Nature methods 9 (7) (2012) 671-675.

[42] G. Bradski, The opencv library., Dr. Dobb's Journal: Software Tools for the Professional Programmer 25 (11) (2000) 120-123.

[43] U. Schmidt, M. Weigert, C. Broaddus, G. Myers, Cell detection with starconvex polygons, in: International Conference on Medical Image Computing and Computer-Assisted Intervention, Springer, 2018, pp. 265-273. 
[44] O. Ronneberger, P. Fischer, T. Brox, U-net: Convolutional networks for biomedical image segmentation, in: International Conference on Medical image computing and computer-assisted intervention, Springer, 2015, pp. 234-241.

[45] U. Phromsuwan, C. Sirisathitkul, Y. Sirisathitkul, B. Uyyanonvara, P. Muneesawang, Application of image processing to determine size distribution of magnetic nanoparticles, Journal of Magnetics 18 (3) (2013) 311-316.

[46] P. Bele, F. Jager, U. Stimming, Image processing for tem micrographs of platinum nanoparticles on glassy carbon, Microscopy and Analysis 122 (2007) S5.

[47] S. Mondini, A. M. Ferretti, A. Puglisi, A. Ponti, Pebbles and pebblejuggler: software for accurate, unbiased, and fast measurement and analysis of nanoparticle morphology from transmission electron microscopy (tem) micrographs, Nanoscale 4 (17) (2012) 5356-5372.

[48] K. He, X. Zhang, S. Ren, J. Sun, Deep residual learning for image recognition, in: Proceedings of the IEEE conference on computer vision and pattern recognition, 2016, pp. 770-778.

[49] Y. Guo, Y. Liu, A. Oerlemans, S. Lao, S. Wu, M. S. Lew, Deep learning for visual understanding: A review, Neurocomputing 187 (2016) 27-48.

[50] L. von Chamier, R. F. Laine, J. Jukkala, C. Spahn, D. Krentzel, E. Nehme, M. Lerche, S. Hernández-Pérez, P. K. Mattila, E. Karinou, et al., Democratising deep learning for microscopy with zerocostdl4mic, Nature communications 12 (1) (2021) 1-18.

[51] M. J. Eslamibidgoli, F. Tipp, J. Jitsev, M. Jankovic, J Eikerling, K. Malek, Convolutional neural networks for high throughput screening of catalyst layer inks for polymer electrolyte fuel cells, RSC Advances 11 (2021) 3212632134 . 
[52] L. Yao, Z. Ou, B. Luo, C. Xu, Q. Chen, Machine learning to reveal nanoparticle dynamics from liquid-phase tem videos, ACS central science 6 (8) (2020) 1421-1430.

${ }_{445}$ [53] K. T. Mukaddem, E. J. Beard, B. Yildirim, J. M. Cole, Imagedataextractor: A tool to extract and quantify data from microscopy images, Journal of chemical information and modeling 60 (5) (2019) 2492-2509.

[54] B. Yildirim, J. M. Cole, Bayesian particle instance segmentation for electron microscopy image quantification, Journal of Chemical Information and Modeling 61 (3) (2021) 1136-1149.

[55] P. Bankhead, M. B. Loughrey, J. A. Fernández, Y. Dombrowski, D. G. McArt, P. D. Dunne, S. McQuaid, R. T. Gray, L. J. Murray, H. G. Coleman, et al., Qupath: Open source software for digital pathology image analysis, Scientific reports 7 (1) (2017) 1-7.

[56] E. Upschulte, S. Harmeling, K. Amunts, T. Dickscheid, Contour proposal networks for biomedical instance segmentation, arXiv preprint arXiv:2104.03393.

[57] E. Xie, W. Wang, M. Ding, R. Zhang, P. Luo, Polarmask++: Enhanced polar representation for single-shot instance segmentation and beyond, IEEE Transactions on Pattern Analysis and Machine Intelligence.

[58] D. W. Scott, Sturges' rule, Wiley Interdisciplinary Reviews: Computational Statistics 1 (3) (2009) 303-306.

[59] D. W. Scott, Scott's rule, Wiley Interdisciplinary Reviews: Computational Statistics 2 (4) (2010) 497-502.

[60] R. Sahann, T. Möller, J. Schmidt, Histogram binning revisited with a focus on human perception, arXiv preprint arXiv:2109.06612.

[61] E. Olson, A study of the effects of histogram binning on the accuracy and precision of particle sizing measurements, Pharmaceutical Technology (2018) 29. 
[62] M. Wand, Data-based choice of histogram bin width, The American Statistician 51 (1) (1997) 59-64.

[63] Z. Fang, B. R. Patterson, M. E. Turner, Modeling particle size distributions by the weibull distribution function, Materials Characterization

\section{4}

[64] S. Baker, R. D. Cousins, Clarification of the use of chi-square and likelihood functions in fits to histograms, Nuclear Instruments and Methods in Physics Research 221 (2) (1984) 437-442.

[65] S.-H. Lin, Y.-M. Yeh, B. Chen, Exploiting polynomial-fit histogram equalization and temporal average for robust speech recognition., in: Interspeech, 2006.

[66] A. Abd-Elfattah, Goodness of fit test for the generalized rayleigh distribution with unknown parameters, Journal of Statistical Computation and Simulation 81 (3) (2011) 357-366.

[67] B. P. MacLeod, F. G. Parlane, T. D. Morrissey, F. Häse, L. M. Roch, K. E. Dettelbach, R. Moreira, L. P. Yunker, M. B. Rooney, J. R. Deeth, et al., Self-driving laboratory for accelerated discovery of thin-film materials, Science Advances 6 (20) (2020) eaaz8867.

[68] B. Burger, P. M. Maffettone, V. V. Gusev, C. M. Aitchison, Y. Bai, X. Wang, X. Li, B. M. Alston, B. Li, R. Clowes, et al., A mobile robotic chemist, Nature 583 (7815) (2020) 237-241.

[69] R. Shimizu, S. Kobayashi, Y. Watanabe, Y. Ando, T. Hitosugi, Autonomous materials synthesis by machine learning and robotics, APL Materials 8 (11) (2020) 111110 . 
[70] C. W. Coley, N. S. Eyke, K. F. Jensen, Autonomous discovery in the chemical sciences part i: Progress, Angewandte Chemie International Edition 59 (51) (2020) 22858-22893.

[71] A. Malek, M. J. Eslamibidgoli, M. Mokhtari, Q. Wang, M. H. Eikerling, K. Malek, Virtual materials intelligence for design and discovery of advanced electrocatalysts, ChemPhysChem 20 (22) (2019) 2946-2955.

[72] D. Krause, Juwels: Modular tier-0/1 supercomputer at the jülich supercomputing centre, Journal of Large-scale Research Facilities, JLSRF 5 (2019) 135. 